\title{
RADON-NIKODYM THEOREMS FOR MULTIMEASURES AND TRANSITION MULTIMEASURES
}

\author{
NIKOLAOS S. PAPAGEORGIOU
}

(Communicated by R. Daniel Mauldin)

\begin{abstract}
In this paper we prove two set valued Radon-Nikodym theorems. One for simple multimeasures and the other for transition multimeasures. In the process of proving the second theorem, we obtain a result concerning transition selectors of transition multimeasures, which is of independent interest.
\end{abstract}

\section{INTRODUCTION}

The study of set valued measures (multimeasures) was first motivated by the needs of mathematical economics and in particular the search of equilibria in exchange economies with production, in which coalitions are the primary economic units. We refer to the works of Vind [21] and Debreu-Schmeidler [7] for more details. Since then the subject of multimeasures has been developed extensively. Important contributions were made, among others, by Artstein [1], Costé [4, 5], Costé-Pallu de la Barriere [6], Debreu-Schmeidler [7], Drewnowski [10], Godet-Thobie [12], Hiai [14] and Pallu de la Barriere [17].

One of the major problems in the theory of multimeasures is that of the existence of set valued Radon-Nikodym derivatives. This issue was first considered by Debreu-Schmeidler [7] and Artstein [1] for $\mathbf{R}^{n}$-valued multimeasures. Then it was extended to Banach space valued multimeasures by Costé [5], Costé-Pallu de la Barriere [6] and Hiai [14].

The purpose of this note is to prove two such set valued versions of the Radon-Nikodym theorem. One for simple multimeasures and the other for multimeasures parameterized by the elements of a measure space (transition multimeasures). The first result is along the lines of those by Coste [5], CostéPallu de la Barriere [6] and Hiai [14], but with different hypotheses. The second result appears to be the first of its kind in the literature. Furthermore, in the process of its proof we obtain some other results concerning transition multimeasures, which are actually of independent interest.

Received by the editors November 6, 1989.

1980 Mathematics Subject Classification (1985 Revision). Primary 46G10, 28 B20.

Key words and phrases. Multimeasure, transition multimeasure, vector measure, bounded variation, Radon-Nikodym property, selection theorem, measurable multifunctions.

Research supported by N. S. F. Grant DMS-8802688. 
Closing this introductory section, we would like to mention that transition multimeasures are an important tool in the study of Markov temporary equilibrium processes in dynamic economies (see Blume [3]).

\section{Preliminaries}

In this section we fix our notation and terminology and briefly recall some basic facts from the theories of multifunctions and multimeasures that will be needed in the sequel.

Let $(\Omega, \Sigma)$ be a measurable space and $X$ a separable Banach space. We will be using the following notations:

$$
P_{f(c)}(X)=\{A \subseteq X: \text { nonempty, closed, (convex) }\}
$$

and

$$
P_{w k c}(X)=\{A \subseteq X \text { : nonempty, } w \text {-compact, convex }\} .
$$

Also, by $X_{w^{*}}^{*}$, we will denote the dual of $X$ endowed with the $w^{*}$-topology and, by $P_{k c}\left(X_{w^{*}}^{*}\right)$, we will denote the nonempty, $w^{*}$-compact and convex subsets of $X^{*}$.

If $A \in 2^{X} \backslash\{\varnothing\}$, we set $|A|=\sup \{\|x\|: x \in A\}$ (the "norm" of the set $A$ ), $\sigma\left(x^{*}, A\right)=\sup \left\{\left(x^{*}, x\right): x \in A\right\}, x^{*} \in X^{*}$ (the "support function" of the set $A$ ) and $d(z, A)=\inf \{\|z-x\|: x \in A\}$ (the "distance function" from the set $A)$.

A multifunction $F: \Omega \rightarrow P_{f}(X)$ is said to be measurable, if, for all $z \in X$, the $\mathbf{R}_{+}$-valued function $\omega \rightarrow d(z, F(\omega))$ is measurable. This is equivalent to saying that there exists a sequence of measurable functions $f_{n}: \Omega \rightarrow X, n \geq 1$ such that $F(\omega)=\operatorname{cl}\left\{f_{n}(\omega)\right\}_{n>1}$ for all $\omega \in \Omega$. Furthermore, if there exists a complete $\sigma$-finite measure $\mu(\cdot)$ on $(\Omega, \Sigma)$, then the above definitions are also equivalent to saying that $\operatorname{Gr} F=\{(\omega, x): x \in F(\omega)\} \in \Sigma \times B(X)$, with $B(X)$ being the Borel $\sigma$-field of $X$ (graph measurability). For further details we refer to Wagner [22, Theorem 4.2].

We define $S_{F}^{1}=\left\{f(\cdot) \in L^{1}(X): f(\omega) \in F(\omega) \mu\right.$-a.e. $\}$, i.e., $S_{F}^{1}$ is the set of all Bochner integrable selectors of $F(\cdot)$. This set may be empty. It is easy to check, using Aumann's selection theorem, that it is nonempty if and only if $\omega \rightarrow \inf \{\|x\|: x \in F(\omega)\} \in L_{+}^{1}$. We will say that $F(\cdot)$ is integrably bounded if $F(\cdot)$ is measurable and $\omega \rightarrow|F(\omega)|$ belongs in $L_{+}^{1}$. In this case, clearly, $S_{F}^{1} \neq \varnothing$. Using $S_{F}^{1}$ we can define a set valued integral for $F(\cdot)$ by setting $\int_{\Omega} F(\omega) d \mu(\omega)=\left\{\int_{\Omega} f(\omega) d \mu(\omega): f \in S_{F}^{1}\right\}$.

Next let $X$ be a Banach space. A multimeasure is a map $M: \Sigma \rightarrow 2^{X} \backslash\{\varnothing\}$ such that (i) $M(\varnothing)=\{0\}$ and (ii) for every sequence $\left\{A_{n}\right\}_{n \geq 1}$ of pairwise disjoint $\Sigma$-sets we have $M\left(\cup_{n \geq 1} A_{n}\right)=\sum_{n \geq 1} M\left(A_{n}\right)$. Depending on the way we interpret this equality of sets, we get different types of multimeasures. However, they all coincide when $M(\cdot)$ is $P_{w k c}(X)$-valued (see Proposition 3 of GodetThobie [12] and Pallu de la Barriere [17]). This fact is the set valued analogue of the well known Orlicz-Pettis theorem (see, for example, Diestel-Uhl [8]). So 
for the needs of this paper we can say that a multivalued set function $M: \Sigma \rightarrow$ $P_{f}(X)$ is a multimeasure if and only if, for all $x^{*} \in X^{*}, A \rightarrow \sigma\left(x^{*}, M(A)\right)$ is a signed measure. Similarly $M: \Sigma \rightarrow P_{k c}\left(X_{w^{*}}^{*}\right)$ is an $X_{w^{*}}^{*}$-valued multimeasure if and only if, for all $x \in X, A \rightarrow \sigma(x, M(A))$ is a signed measure.

For a set valued measure $M(\cdot)$ and for $A \in \Sigma$, we set

$$
|M|(A)=\sup _{\pi} \sum_{k}\left|M\left(A_{k}\right)\right|,
$$

where the supremum is taken over all finite $\Sigma$-partitions $\pi=\left\{A_{k}\right\}_{k=1}^{n}$ of $A$. It is easy to check that $|M|(\cdot)$ is a measure. If $|M|(\Omega)<\infty$, then we say that the multimeasure $M(\cdot)$ is of bounded variation. We say that the multimeasure $M(\cdot)$ is absolutely continuous with respect to the measure $\mu(\cdot)$, denoted by $M \ll \mu$, if for all $A \in \Sigma$ with $\mu(A)=0 \Rightarrow M(A)=\{0\}$. Every vector measure $m: \Sigma \rightarrow X$ satisfying $m(A) \in M(A)$ for all $A \in \Sigma$ is said to be a selector of $M(\cdot)$. We denote the set of all selectors of $M(\cdot)$ by $S_{M}$. On $S_{M}$ we can define the topology $\tau$ of simple weak convergence (i.e., $m_{a} \stackrel{\tau}{\longrightarrow} m$ if and only if, for all $\left.A \in \Sigma, m_{a}(A) \stackrel{w}{\longrightarrow} m(A)\right)$, as follows. Let $S$ be the space of all $\mathbf{R}$-valued simple functions, with a finite number of values. Consider $S \otimes X^{*}$. Then the space $c a(X)$ of all $X$-valued vector measures and $S \otimes X^{*}$ can be put in duality by

$$
\langle m, u\rangle=\left\langle m, \sum_{k=1}^{n} \chi_{A_{k}} \otimes x_{k}^{*}\right\rangle=\sum_{k=1}^{n}\left(x_{k}^{*}, m\left(A_{k}\right)\right),
$$

where $\left\{A_{k}\right\}_{k=1}^{n}$ is a finite $\Sigma$-partition of $\Omega$ and $x_{k}^{*} \in X^{*}, k=1,2, \ldots, n$. Then it is easy to see that the topology of simple weak convergence on $c a(X)$ is nothing else but the weak topology $w\left(c a(X), S \otimes X^{*}\right)$.

Now let $(\Omega, \Sigma)$ and $(T, \mathscr{T})$ be measurable spaces and $X$ a separable Banach space. A multivalued map $M: \Omega \times \mathscr{T} \rightarrow P_{f}(X)$ is said to be a transition multimeasure if

(i) for all $A \in \mathscr{T}, \omega \rightarrow M(\omega, A)$ is a measurable multifunction,

(ii) for all $\omega \in \Omega, A \rightarrow M(\omega, A)$ is a multimeasure.

A "selector transition measure" or simply a "transition selector" is a map $m: \Omega \times \mathscr{T} \rightarrow X$ such that

(i) $)^{\prime}$ for all $A \in \mathscr{T}, \omega \rightarrow m(\omega, A)$ is $\Sigma$-measurable,

(ii) $^{\prime}$ for all $\omega \in \Omega, A \rightarrow m(\omega, A)$ is a vector measure,

(iii) $^{\prime}$ for all $(\omega, A) \in \Omega \times \mathscr{T}, m(\omega, A) \in M(\omega, A)$.

The set of all transition selectors of $M(\cdot, \cdot)$ will be denoted by $T S_{M}$. Similarly we can define a $P_{k c}\left(X_{w^{*}}^{*}\right)$-valued multimeasure and its set of transition selectors.

Let $T$ be a Polish space and $X$ a Banach space. By $C_{b}(T)$ we will denote the space of bounded, continuous functions on $T$ and by $C_{b}(T) \otimes X$ the space of bounded continuous functions with values in a finite dimensional subspace of $X$. Also, by $c a_{b}(T, X)$, we will denote the space of all $X$-valued vector 
measures of bounded variation defined on $(T, B(T))$. Similarly we define $c a_{b}\left(T, X_{w^{*}}^{*}\right)$ and $C_{b}(T) \otimes X^{*}$.

In the sequel all measure spaces are assumed to be positive.

\section{RADON-NIKODYM THEOREM FOR MULTIMEASURES}

In this section we present a new Radon-Nikodym theorem for $P_{w k c}(X)$ valued multimeasures. Note that, contrary to Costé [5, Theorem 3] and Hiai [14, Theorem 4.5], we make no hypotheses on the structure of $X^{*}$, and instead we strengthen our hypotheses on $X$. Also compared to Theorem 3.1 of CostePallu de la Barriere [6], we see that we do not need the strong boundedness hypothesis that the authors had in that theorem.

Theorem 3.1. If $(\Omega, \Sigma, \mu)$ is a finite positive measure space, $X$ a weakly sequentially complete Banach space with the Radon-Nikodym property $(R N P)$ such that $l^{1} \leftrightarrow X$ and $M: \Sigma \rightarrow P_{w k c}(X)$ is a multimeasure of bounded variation such that $M \ll \mu$, then there exists $F: \Omega \rightarrow P_{w k c}(X)$ integrably bounded such that, for all $A \in \Sigma$, we have

$$
M(A)=\int_{A} F(\omega) d \mu(\omega) .
$$

Proof. From Theorem 1(i) of Godet-Thobie [12] we know that, for every $A \in$ $\Sigma$, we have

$$
M(A)=\left\{m(A): m \in S_{M}\right\} .
$$

Since by hypothesis $X$ has the RNP and $m(\cdot)$ is a $\mu$-continuous vector measure of bounded variation, there exists $\frac{d m}{d \mu}(\cdot)=f_{m}(\cdot) \in L^{1}(X)$. Set $K=$ $\left\{f_{m}(\cdot) \in L^{1}(X): m \in S_{M}\right\}$. Observe that, for every $(A, m) \in \Sigma \times S_{M}$ we have $\int_{A}\left\|f_{m}(\omega)\right\| d \mu(\omega) \leq|M|(A)$. So $K$ is uniformly integrable and since $l^{1} \nrightarrow X$, from Pisier [18], we know that $K$ has no $l^{1}$-subsequence. Hence proposition IV of Assani [2] tells us that $K$ is relatively $w$-compact in $L^{1}(X)$. We claim that $K$ is $w$-closed. So let $f_{m_{a}} \stackrel{w}{\longrightarrow} f$ in $L^{1}(X)$. From Theorem 1(ii) of Godet-Thobie [12], we know that $S_{M}$ is $w\left(c a(X), S \otimes X^{*}\right)$-compact. So, by passing to a subnet if necessary, we may assume that $m_{a} \rightarrow m \in S_{M}$ in $w\left(c a(X), S \otimes X^{*}\right)$. So, for all $\left(A, x^{*}\right) \in \Sigma \times X^{*}, \int_{\Omega}\left(\chi_{A}(\omega) x^{*}, f_{m_{a}}(\omega)\right) d \mu(\omega) \rightarrow$ $\int_{\Omega}\left(\chi_{A}(\omega) x^{*}, f_{m}(\omega)\right) d \mu(\omega) \Rightarrow \int_{A}\left(x^{*}, f_{m}(\omega)\right) d \mu(\omega)=\int_{A}\left(x^{*}, f(w)\right) d \mu(w)$ for all $\left(A, x^{*}\right) \in \Sigma \times X^{*}$. Let $\left\{x_{n}^{*}\right\}_{n \geq 1}$ be $w^{*}$-dense in $X^{*}$. Such a countable set exists since $X$ is separable (see, for example, Wilansky [23, p. 144]). Then we have $\left(x_{n}^{*}, f_{m}(\omega)\right)=\left(x_{n}^{*}, f(\omega)\right)$ for all $\omega \in \Omega \backslash N, \mu(N)=0$ and all $n \geq 1$, and this, by the $w^{*}$-density of the $x_{n}^{*}$ 's in $X^{*}$, implies that $\left(x^{*}, f_{m}(\omega)\right)=$ $\left(x^{*}, f(\omega)\right)$ for all $\omega \in \Omega \backslash N$ and ail $x^{*} \in X^{*} \Rightarrow f_{m}(\omega)=f(\omega) \mu$-a.e. Thus $K$ is $w$-closed, hence $w$-compact in $L^{1}(X)$ and of course convex. Invoking Theorem 4 of Klei [15], we deduce that there exists $F: \Omega \rightarrow P_{w k c}(X)$ integrably bounded such that $K=S_{F}^{1}$. Therefore, for all $A \in \Sigma$, we have $M(A)=$ $\int_{A} F(\omega) d \mu(\omega)$. 
Remark. It is well known that a separable Banach space with no $l^{1}$-copy need not have a separable dual (recall the James Tree Space, see Diestel-Uhl [8, p. 214]). So we have two different classes of spaces: those for which $l^{1} \nrightarrow X$ and those with dual that has the RNP (Asplund spaces). In [19], Riddle-Uhl showed that the difference between these two classes of spaces can be expressed in terms of martingales.

\section{RADON-NIKODYM THEOREM FOR TRANSITION MULTIMEASURES}

In this section we present a Radon-Nikodym theorem for transition multimeasures. In order to do this, we will need two results about transition measures and multimeasures that are actually of independent interest.

Proposition 4.1. If $(\Omega, \Sigma, \mu)$ is a complete $\sigma$-finite measure space, $T$ is a Polish space with a $\sigma$-finite measure $\lambda(\cdot)$ on $B(T)=$ the Borel $\sigma$-field of $T, X$ is a separable reflexive Banach space and $m: \Omega \times B(T) \rightarrow X$ a transition vector measure such that $m(\omega, \cdot) \ll \lambda \mu$-a.e. and $|m(\omega, \cdot)| \leq \phi(\omega) \mu$-a.e. with $\phi(\cdot) \in L_{+}^{1}(\Omega)$ (here $|m(\omega, \cdot)|$ denotes the variation of the vector measure $m(\omega, \cdot))$, then there exists $f: \Omega \times T \rightarrow X$ measurable function and $N \in \Sigma$ with $\mu(N)=0$ such that $f(\omega, \cdot) \in L^{1}(T, \lambda, X)$ for every $\omega \in \Omega$ and $m(\omega, C)=\int_{C} f(\omega, t) \lambda(d t)$ for all $\omega \in \Omega \backslash N$ and $C \in B(T)$.

Proof. Since, by hypothesis, $m(\omega, \cdot)$ is of bounded variation, $m(\omega, \cdot) \ll \lambda$ for all $\omega \in \Omega \backslash N_{1}, \mu\left(N_{1}\right)=0$ and $X$ is reflexive (hence it has the RNP), for $\omega \in \Omega \backslash N_{1}$ there exists $f(\omega, \cdot) \in L^{1}(T, \lambda, X)$ such that $m(\omega, C)=$ $\int_{C} f(\omega, t) \lambda(d t)$ and $\|f(\omega, \cdot)\|_{L^{1}(T, \lambda, x)} \leq \phi(\omega) \mu$-a.e. By redefining $\omega \rightarrow f(\omega, \cdot)$ on $N_{1}$ we may assume that $f(\omega, \cdot) \in L^{1}(T, \lambda, X)$ for all $\omega \in \Omega$. Then, for every $\left(x^{*}, C\right) \in X^{*} \times B(T)$,

$$
\left\langle f(\omega, \cdot), \chi_{C} x^{*}\right\rangle=\left(x^{*}, m(\omega, C)\right),
$$

where $\langle\cdot, \cdot\rangle$ denotes the duality brackets for the pair

$$
\left(L^{1}(T, \lambda, X), L^{\infty}\left(T, \lambda, X^{*}\right)\right)
$$

(recall $\left(L^{1}(T, \lambda, X)\right)^{*}=L^{\infty}\left(T, \lambda, X^{*}\right)$, see Diestel-Uhl [8, p. 98]). Hence $\omega \rightarrow\left\langle f(\omega, \cdot), \chi_{C} x^{*}\right\rangle$ is measurable. Since countably valued functions are dense in $L^{\infty}\left(T, \lambda, X^{*}\right)$ (see Corollary 3, p. 42 of Diestel-Uhl [8]), we deduce that $\omega \rightarrow\langle f(\omega, \cdot), u\rangle$ is measurable for all $u(\cdot) \in L^{\infty}\left(T, \lambda, X^{*}\right) \Rightarrow \omega \rightarrow f(\omega, \cdot)$ is weakly measurable from $\Omega$ into $L^{1}\left(T, \lambda, X^{*}\right)$, and, since the latter is separable, from the Pettis measurability theorem (see Diestel-Uhl [8, p. 42]), we have that $\omega \rightarrow f(\omega, \cdot)$ is measurable from $\Omega$ into $L^{1}(T, \lambda, X)$. Recalling that $\|f(\omega, \cdot)\|_{L^{1}(T, \lambda, x)} \leq \phi(\omega) \mu$-a.e. and $\phi(\cdot) \in L_{+}^{1}(\Omega)$, we get that $\omega \rightarrow f(\omega, \cdot)$ belongs in $L^{1}\left(\Omega, L^{1}(T, \lambda, X)\right)$. Invoking Lemma 16 of Dunford-Schwartz $\left[11\right.$, p. 196] we can find $f(\cdot, \cdot) \in L^{1}(\Omega \times T, \mu \times \lambda, X)$ such that $m(\omega, C)=$ $\int_{C} f(\omega, t) \lambda(d t)$ for all $\omega \in \Omega \backslash N$ and all $C \in B(T)$. 
The next result establishes the existence of a transition selector for a class of transition multimeasures. Our result extends to transition multimeasures, Theorem 2.3 of Hiai [14], and also extends Theorem 5 of Godet-Thobie [12].

Proposition 4.2. If $(\Omega, \Sigma, \mu)$ is a complete, $\sigma$-finite measure space, $T$ a Polish space, $X$ a separable Banach space, $M: \Omega \times B(T) \rightarrow P_{k c}\left(X_{w^{*}}^{*}\right)$ is a transition multimeasure which is of bounded variation for every $\omega \in \Omega$ and $h: \Omega \rightarrow X_{w^{*}}^{*}$ is a measurable map such that, for some $A \in B(T), h(\omega) \in M(\omega, A)$ for all $\omega \in \Omega$, then there exists $m \in T S_{M}$ such that, for all $\omega \in \Omega, m(\omega, A)=h(\omega)$.

Proof. Let $R_{A}: \Omega \rightarrow 2^{c a_{b}\left(T, X_{w^{*}}^{*}\right)}$ be defined by

$$
R_{A}(\omega)=\left\{m \in c a_{b}\left(T, X_{w^{*}}^{*}\right): m \in S_{M(\omega, \cdot)}, m(A)=h(\omega)\right\} .
$$

From Theorem 1 of Godet-Thobie [12] (see also Theorem 2.3 of Hiai [14]), we know that, for all $\omega \in \Omega, R_{A}(\omega) \neq \varnothing$.

Next let $x \in X$ and consider the function $\phi_{A, x}: \Omega \times c a_{b}\left(T, X_{w^{*}}^{*}\right) \rightarrow \mathbf{R}$ defined by $\phi_{A, x}(\omega, m)=(x, m(A)-h(\omega))$. Since, by hypothesis, $h(\cdot)$ is $w^{*}$ measurable, $\omega \rightarrow(x, h(\omega))$ is a measurable $\mathbf{R}$-valued function. On the other hand recall that, by the definition of $C_{b}(T) \otimes X$, the $w\left(c a_{b}\left(T, X_{w^{*}}^{*}\right), C_{b}(T) \otimes X\right)$ topology is the weakest topology for which $m \rightarrow x o m$ is continuous from $c a_{b}\left(T, X_{w^{*}}^{*}\right)$ into $c a_{b}(T)$ with the weak (narrow) topology (here $x o m(\cdot)$ denotes the R-valued measure $A \rightarrow(x, m(A)))$. Also from the Dynkin system theorem, we deduce that for every $C \in B(T)$, the map $\lambda \rightarrow \lambda(C)$ on $c a_{b}(T)$ with the weak topology is measurable. Therefore we see that $(\omega, m) \rightarrow$ $(x, m(A))-(x, h(\omega))=\phi_{A, x}(\omega, m)$ is jointly measurable on $\Omega \times c a_{b}\left(T, X_{w^{*}}^{*}\right)$, when $c a_{b}\left(T, X_{w^{*}}^{*}\right)$ is endowed with the $w\left(c a_{b}\left(T, X_{w^{*}}^{*}\right), C_{b}(T) \otimes X\right)$-topology.

By definition $m(\cdot)$ is a measure selector of $M(\omega, \cdot)$ (denoted by $m(\cdot) \in$ $\left.S_{M(\omega, \cdot)}\right)$ if and only if, for all $C \in B(T), m(C) \in M(\omega, C)$. Since $M(\cdot, \cdot)$ is $P_{k c}\left(X_{w^{*}}^{*}\right)$-valued, we have $(x, m(C)) \leq \sigma(x, M(\omega, C))$ for all $x \in X$ and all $C \in B(T)$. Note that, since $M(\cdot, \cdot)$ is a transition multimeasure, $\omega \rightarrow$ $\sigma(x, M(\omega, C))$ is measurable, while, as above, we can show $m \rightarrow(x, m(C))$ is measurable from $c a_{b}\left(T, X_{w^{*}}^{*}\right)$ with the $w\left(c a_{b}\left(T, X_{w^{*}}^{*}\right), C_{b}(T) \otimes X\right)$-topology into $\mathbf{R}$. Hence the map

$$
\eta_{C, x}(\omega, m)=\sigma(x, M(\omega, C))-(x, m(C))
$$

is jointly measurable.

Now let $\left\{x_{k}\right\}_{k \geq 1}$ be dense in $X$ and let $\left\{C_{n}\right\}_{n \geq 1}$ be a field generating $B(T)$, i.e., $\sigma\left(\left\{C_{n}\right\}_{n \geq 1}\right)=B(T)$ (recall that, since $T$ is a Polish space, $B(T)$ is countably generated and so such a field exists). Then, by setting $\phi_{A, k}(\omega, m)=$ $\left(x_{k}, m(A)-h(\omega)\right)$ and $\eta_{n, k}(\omega, m)=\sigma\left(x_{k}, M\left(\omega, C_{n}\right)\right)-\left(x_{k}, m\left(C_{n}\right)\right)$, we can write

$$
\begin{aligned}
\operatorname{Gr} R_{A}= & \bigcap_{\substack{k \geq 1 \\
n \geq 1}}\left\{(\omega, m) \in \Omega \times c a_{b}\left(T, X_{w^{*}}^{*}\right): \phi_{A, k}(\omega, m)=0, \eta_{n, k}(\omega, m) \geq 0\right\} \\
& \in \Sigma \times B\left(c a_{b}\left(T, X_{w^{*}}^{*}\right)\right) .
\end{aligned}
$$


From Theorem 3, p. 337 of Saint-Beuve [20], we know that $c a_{b}\left(T, X_{w^{*}}^{*}\right)$, equipped with the $w\left(c a_{b}\left(T, X_{w^{*}}^{*}\right), C_{b}(T) \otimes X\right)$-topology, is a Souslin space. Hence we can apply Aumann's selection theorem (see Wagner [22, Theorem 5.10]) and get $r: \Omega \rightarrow c a_{b}\left(T, X_{w^{*}}^{*}\right)$ measurable such that $r(\omega) \in R_{A}(\omega)$ for all $\omega \in \Omega$. Set $r(\omega)(C)=m(\omega, C)$ for all $(\omega, C) \in \Omega \times B(T)$. Then clearly $m \in T S_{M}$ and, for all $\omega \in \Omega, m(\omega, A)=h(\omega)$.

Now we are ready to state and prove the Radon-Nikodym theorem for transition multimeasures. By $C\left(T, X^{*}\right)$ we will denote the Banach space of $X^{*}$ valued continuous functions defined on $T$.

Theorem 4.1. If $(\Omega, \Sigma, \mu)$ is a complete $\sigma$-finite measure space, $T$ a compact Polish space with a $\sigma$-finite measure $\lambda(\cdot), X$ a separable reflexive Banach space and $M: \Omega \times B(T) \rightarrow P_{w k c}(X)$ a transition multimeasure which is of bounded variation for every $\omega \in \Omega,|M(\omega, \cdot)| \leq \phi(\omega)$ with $\phi(\cdot) \in L_{+}^{1}$ and $M(\omega, \cdot) \ll \lambda$ for all $\omega \in \Omega$, then there exists $F: \Omega \times T \rightarrow P_{w k c}(X)$ a measurable multifunction such that, for all $\omega \in \Omega, F(\omega, \cdot)$ is integrably bounded and, for all $(\omega, A) \in \Omega \backslash N^{\prime} \times B(T), \mu\left(N^{\prime}\right)=0, M(\omega, A)=\int_{A} F(\omega, t) \lambda(d t)$.

Proof. From Theorem 1 of Godet-Thobie [12] we know that, for every $\omega \in \Omega$, $S_{M(\omega, \cdot)} \neq \varnothing$. Also note that $S_{M(\omega, \cdot)} \subseteq c a_{b}^{\leq|M(\omega, \cdot)|}(T, X)=\left\{m \in c a_{b}(T, X)\right.$ : $|m|(\Omega) \leq|M(\omega, \cdot)|(\Omega)\}$. The latter is an equicontinuous subset of $c a_{b}(T, X)=$ $\left[C\left(T, X^{*}\right)\right]^{*}$ (see, for example, Dinculeanu [9] and recall that, since $T$ is a Polish space, every bounded vector measure is regular) and so, by Ascoli's theorem, on $c a_{b}^{\leq|M(\omega, \cdot)|}(T, X)$ the $w_{1}=w\left(c a_{b}(T, X), C(T) \otimes X^{*}\right)$ and $w_{1}^{\prime}=$ $w\left(c a_{b}(T, X), C\left(T, X^{*}\right)\right)$ topologies coincide (of course in general we have $\left.w_{1} \subseteq w_{1}^{\prime}\right)$. Let $m \in S_{M(\omega, \cdot)}$. Since $m \ll \lambda$ and $X$ is reflexive (hence it has the RNP), there exists $f_{m}(\cdot)=\frac{d m}{d \lambda}(\cdot) \in L^{1}(T, X)$ such that $m(A)=$ $\int_{A} f_{m}(t) d \lambda(t)$. Let $K=\left\{f_{m}(\cdot): m \in S_{M(\omega, \cdot)}\right\} \subseteq L^{1}(T, X)$. Recall that $V_{\mu}=\left\{m \in c a_{b}(T, X): m \ll \mu\right\}$ with the total variation norm is isometrically isomorphic to $L^{1}(T, X)$. Hence $K$ is relatively weakly compact in $L^{1}(T, X)$ if and only if $S_{M(\omega, \cdot)}$ is in $V_{\mu} \subseteq c a_{b}(T, X)$. The latter is relatively $w$-compact, because $S_{M(\omega, \cdot)}$ is a subset of the $w_{1}=w_{1}^{\prime}$-compact set $c a_{b}^{\leq|M(\omega, \cdot)|}(T, X)$. Now let $\left\{m_{n}\right\}_{n \geq 1} \subseteq S_{M(\omega, \cdot)}$ and assume $m_{n} \stackrel{w_{1}=w_{1}^{\prime}}{\longrightarrow} m$. We just saw that $\left\{f_{n}=d m_{n} / d \lambda\right\}_{n \geq 1} \subseteq L^{1}(T, X)$ is relatively weakly compact. So, by passing to a subsequence if necessary, we may assume that $f_{n} \stackrel{w}{\longrightarrow} f$ in $L^{1}(T, X)$. Hence $m_{n}(A)=\int_{A} f_{n}(t) d \lambda(t) \rightarrow \int_{A} f(t) d \lambda(t)=m(A) \in M(\omega, A)$ for all $A \in B(T) \Rightarrow m \in S_{M(\omega, \cdot)}$. Since the $w_{1}=w_{1}^{\prime}$-topology on $V_{\mu}$ is metrizable (see, for example, Dunford-Schwartz [11, Theorem 1, p. 426]), we conclude that $S_{M(\omega, \cdot)}$ is $w_{1}$-closed, hence $w_{1}$-compact.

Now let $R: \Omega \rightarrow P_{k c}\left(c a_{b}(T, X)_{w_{1}}\right)$ be the multifunction defined by

$$
R(\omega)=\left\{m \in c a_{b}(T, X): m(A) \in M(\omega, A) \text { for all } A \in B(T)\right\} .
$$


Since $T$ is a Polish space, $B(T)$ is countably generated. So we can find a field $\left\{A_{n}\right\}_{n \geq 1}$ such that $\sigma\left(\left\{A_{n}\right\}_{n \geq 1}\right)=B(T)$. Also let $\left\{x_{k}^{*}\right\}_{k \geq 1}$ be dense in $X^{*}$. Then

$$
\begin{aligned}
& R(\omega)=\bigcap_{\substack{k \geq 1 \\
n \geq 1}}\left\{x \in c a_{b}(T, X):\left(x_{k}^{*}, m\left(A_{n}\right)\right) \leq \sigma\left(x_{k}^{*}, M\left(\omega, A_{n}\right)\right)\right\} \\
& \Rightarrow \operatorname{Gr} R=\bigcap_{\substack{k \geq 1 \\
n \geq 1}}\left\{(\omega, x) \in \Omega \times c a_{b}(T, X):\left(x_{k}^{*}, m\left(A_{n}\right)\right) \leq \sigma\left(x_{k}^{*}, M\left(\omega, A_{n}\right)\right)\right\} .
\end{aligned}
$$

Recall that, for every $k \geq 1$ and every $n \geq 1, m \rightarrow\left(x_{k}^{*}, m\left(A_{n}\right)\right)$ is measurable from $c a_{b}(T, X)_{w_{1}}$ into $\mathbf{R}$, while $\left.\omega \rightarrow \sigma\left(x_{k}^{*}, M\left(\omega, A_{n}\right)\right)\right\}$ is measurable since $M(\cdot, \cdot)$ is a transition multimeasure. So $(\omega, m) \rightarrow \sigma\left(x_{k}^{*}, M\left(\omega, A_{n}\right)\right)-$ $\left(x_{k}^{*}, m\left(A_{n}\right)\right)$ is measurable for all $k \geq 1, n \geq 1 \Rightarrow \mathrm{Gr} R \in \Sigma \times B\left(c a_{b}(T, X)_{w_{1}}\right)$. From Theorem 3, p. 337 of Saint-Beuve [20] we know that $c a_{b}(T, X)_{w_{1}}$ is a Souslin space. So Theorem 4.2(f) of Wagner [22] tells us that $R^{-}(K)=\{\omega \in$ $\Omega: R(\omega) \cap C \neq \varnothing\} \in \Sigma$ for all $C \subseteq c a_{b}(T, X)_{w_{1}}$ closed. But then, recalling that a Souslin space is Lindelöf and combining Propositions 5.1.12 and 5.1.14 of Hess [13], we deduce that there exist $m_{n}: \Omega \rightarrow c a_{b}(T, X)_{w_{1}}$ measurable maps such that $R(\omega)=\left\{\overline{m_{n}(\omega)}\right\}_{n \geq 1}^{w_{1}}$.

Next we claim that, for every $n \geq 1$ and every $A \in B(T), \omega \rightarrow m_{n}(\omega, A)$ is measurable. Indeed let $\phi_{1}: \Omega \rightarrow c a_{b}(T, X)_{w}$ be defined by $\phi_{1}(\omega)=m_{n}(\omega, \cdot)$ and $\phi_{2}: c a_{b}(T, X)_{w_{1}} \rightarrow X$ by $\phi_{2}(\hat{m})=\hat{m}(A)$. We already know that $\phi_{2}(\cdot)$ is weakly measurable and, since $X$ is separable, measurable. Also $\phi_{1}(\cdot)$ is measurable by construction. Hence $\phi_{2} \circ \phi_{1}: \Omega \rightarrow X$ is measurable $\Rightarrow \omega \rightarrow$ $\phi_{2}\left(\phi_{1}(\omega)\right)=m_{n}(\omega, A)$ is measurable as claimed.

Now we will show that, for all $A \in B(T)$, we have

$$
M(\omega, A)=\overline{\operatorname{conv}}\left\{m_{n}(\omega, A)\right\}_{n \geq 1}
$$

for almost all $\omega \in \Omega$. Suppose not. Then there exist $\Omega_{1} \subseteq \Omega, \mu\left(\Omega_{1}\right)>0$ and $A \in B(T)$ such that $\overline{\operatorname{conv}}\left\{m_{n}(\omega, A)\right\}_{n \geq 1} \varsubsetneqq M(\omega, A)$ for all $\omega \in \Omega_{1}$. Let $L_{A}: \Omega_{1} \rightarrow 2^{X} \backslash\{\varnothing\}$ be defined by $L_{A}(\omega)=M(\omega, A) \backslash \overline{\operatorname{conv}}\left\{m_{n}(\omega, A)\right\}_{n \geq 1}$. Set $M^{\prime}(\omega, A)=\overline{\operatorname{conv}}\left\{m_{n}(\omega, A)\right\}_{n \geq 1}$. We have

$$
\begin{aligned}
& \operatorname{Gr} L_{A}=\operatorname{Gr} M(\cdot, A) \cap\left[\mathrm{Gr} M^{\prime}(\cdot, A)\right]^{c} \\
& \quad \Rightarrow \operatorname{Gr} L_{A} \in \Sigma_{1} \times B(X) \quad(\text { see Wagner [22]), }
\end{aligned}
$$

where $\Sigma_{1}=\Sigma \cap \Omega_{1}$ (the trace of $\Sigma$ on $\Omega_{1}$ ). Apply Aumann's selection theorem to get $x: \Omega_{1} \rightarrow X$ measurable such that $x(\omega) \in L_{A}(\omega)$ for all $\omega \in \Omega_{1}$. Then, invoking Proposition 4.2, we can find $m \in T S_{M}$ such that $x(\omega)=m(\omega, A)$ for all $\omega \in \Omega_{1}$. Fix $\omega \in \Omega_{1}$. Then, since $R(\omega)=S_{M(\omega, \cdot)}=\left\{\overline{m_{n}(\omega)(\cdot)}\right\}_{n \geq 1}^{w_{1}}$, we can find $\left\{m_{k}(\omega, \cdot)\right\}_{k \geq 1} \subseteq\left\{m_{n}(\omega, \cdot)\right\}_{n \geq 1}$ such that $m_{k}(\omega, \cdot) \stackrel{w_{1}}{\longrightarrow} m(\omega, \cdot)$. As 
in the beginning of the proof, we can get that $m_{k}(\omega, A) \stackrel{w}{\longrightarrow} m(\omega, A)$, a contradiction to the choice of $m(\cdot, \cdot)$. So, indeed, $M(\omega, A)=\overline{\operatorname{conv}}\left\{m_{n}(\omega, A)\right\}_{n \geq 1}$ for all $(\omega, A) \in \Omega \times B(T)$. Apply Proposition 4.1 to get $f_{n}: \Omega \times T \stackrel{\rightarrow}{\rightarrow}$ $X, n \geq 1$ measurable such that, for all $\omega \in \Omega, f_{n}(\omega, \cdot) \in L^{1}(T, \lambda, X)$ and $m_{n}(\omega, A)=\int_{A} f_{n}(\omega, t) \lambda(d t)$ for all $\omega \in \Omega \backslash N, \mu(N)=0$ and all $A \in B(T)$. Set $F(\omega, t)=\overline{\operatorname{conv}}\left\{f_{n}(\omega, t)\right\}_{n>1}$. Then $F: \Omega \times T \rightarrow P_{w k c}(X)$ is jointly measurable and integrably bounded in $t$. Furthermore, from the properties of the set valued integral (see [16]) we have, for every $(\omega, A) \in \Omega \backslash N^{\prime} \times B(T)$, $\mu\left(N^{\prime}\right)=0$

$$
\begin{aligned}
M(\omega, A) & =\overline{\operatorname{conv}}\left\{m_{n}(\omega, A)\right\}_{n \geq 1}=\overline{\operatorname{conv}}\left\{\int_{A} f_{n}(\omega, t) \lambda(d t)\right\}_{n \geq 1} \\
& =\int_{A} \overline{\operatorname{conv}}\left\{f_{n}(\omega, t)\right\}_{n \geq 1} \lambda(d t) \\
& =\int_{A} F(\omega, t) \lambda(d t) .
\end{aligned}
$$

\section{ACKNOWLEDGMENT}

The author is grateful to the referee for his careful reading of the paper and his many corrections and remarks that improved the content considerably.

\section{REFERENCES}

1. Z. Artstein, Set valued measures, Trans. Amer. Math. Soc. 65 (1972), 103-125.

2. I. Assani, Quelques résultas lies aux ensembles décomposables de $L_{E}^{1}$, C. R. Acad. Sci. Paris. 294 (1982), 641-644.

3. L. Blume, New techniques for the study of stochastic equilibrium processes, J. Math. Econom. 9 (1982), 61-70.

4. A. Costé, Sur les multimesures a valeurs fermes bornes d'un espace de Banach, C. R. Acad. Sci. Paris. 280 (1975), 567-570.

5. __ La propriété de Radon-Nikodym en integration multivoque, C. R. Acad. Sci. Paris. 280 (1975), 1515-1518.

6. A. Costé and R. Pallu de la Barriere, Radon-Nikodym theorems for set valued measures whose values are convex and closed, Ann. Soc. Math. Polon. Ser. I Comment. Math. Prace Mat. XX (1978), 283-309.

7. G. Debreu and D. Schmeidler, The Radon-Nikodym derivative of a correspondence, in Proceedings of Sixth Berkeley Sym. Math. Stat. and Probability (L. Le Cam, J. Neymann and E. Scott, eds.), Univ. of California Press, Berkeley, 1972, pp. 41-56.

8. J. Diestel and J. Uhl, Vector measures, Math. Surveys, vol. 15, Amer. Math. Soc., Providence, RI, 1977.

9. N. Dinculeanu, Vector measures, Pergamon Press, London, 1966.

10. L. Drewnowski, Additive and countably additive correspondences, Ann. Soc. Math. Polon. Series I Comment. Math. Prace Mat. XIX (1976), 25-54.

11. N. Dunford and J. Schwartz, Linear operators I, Wiley, New York, 1958.

12. C. Godet-Thobie, Some results about multimeasures and their selectors, Measure Theory-Oberwolfach 1979 (D. Kölzow, ed.), Lecture Notes in Math., vol. 794, Springer, Berlin, $1980,112-116$. 
13. C. Hess, Mesurabilité, convergence et approximation des multifonctions a valeurs dans un e.l.c.s., Sém. Anal. Convexe, exposé 9 (1985), 9.1-9.100.

14. F. Hiai, Radon-Nikodym theorems for set valued measures, J. Multiv. Anal. 8 (1978), 96118.

15. D. Kandilakis and N. S. Papageorgiou, On the properties of the Aumann integral with applications to differential inclusions and control systems, Czechoslovak Math. J. 39 (1989), $1-15$.

16. H.-A. Klei, Compacité faible de parties decomposables de $L_{E}^{1}$, C. R. Acad. Sci. Paris. 296 (1983), 965-967.

17. R. Pallu de la Barriere, Introduction a l'etude des multi-mesures, in Seminaire Initiation a l'Analyse (G. Choquet, M. Rogalski, and J. Saint Raymond, eds.), 19e année 1979/80, no. 7, 7-01-7-23.

18. G. Pisier, Une propriété de stabilité de la classe des espaces ne contenant pas $l^{1}, \mathrm{C} . \mathrm{R}$. Acad. Sci. Paris. 286 (1978), 747-750.

19. L. Riddle and J. Uhl, Martingales and the fine line between Asplund spaces and spaces not containing a copy of $l^{1}$, in Martingale Theory in Harmonic Analysis and Banach Spaces ( $\mathrm{J}$. Chao and W. Woyczynski, eds.), Lecture Notes in Math. vol. 939, Springer, Berlin, 1982, 145-156.

20. M.-F. Saint-Beuve, Some topological properties of vector measures with bounded variations and its applications, Ann. Mat. Pura Appl. CXVI (1978), 317-379.

21. K. Vind, Edgeworth allocations in an exchange economy with many traders, Internat. Econom. Rev. 5 (1964), 165-177.

22. D. Wagner, Survey of measurable selection theorems, SIAM J. Control Optim. 15 (1977), 859-903.

23. A. Wilansky, Modern methods in topological vector spaces, McGraw-Hill, New York, 1978.

1015 Department of Mathematics, University of California, Davis, California 95616 